\title{
ANN, MFCC and its applications in speaker recognition
}

\author{
Salaheddine KHAMLICH ${ }^{1}$, Mohamed ELOURMI' ${ }^{2}$ Abdellah AILANE ${ }^{3}$ \\ Deptt. of Electronics Engg., ENSA KHOURIBGHA, Morocco ${ }^{1}$ \\ Deptt. of Electronics and Telecom., ENSA ELJADIDA, Morocco² \\ Deptt. of Electronics Engg., ENSA KHOURIBGHA, Morocco ${ }^{3}$
}

\begin{abstract}
This paper presents different hardware implementations of a multi-layer perceptron (MLP) and Mel Frequency Cepstrum Coefficient (MFCC) for speech recognition. Speech processing is emerged as one of the important application area of digital signal processing. Various fields for research in speech processing are speech recognition, speaker recognition, speech synthesis, speech coding etc. The objective of automatic speaker recognition is to extract, characterize and recognize the information about speaker identity. Feature extraction is the first step for speaker recognition. Many algorithms are suggested/developed by the researchers for feature extraction. In this work, the Mel Frequency Cepstrum Coefficient (MFCC) feature has been used for designing a text dependent speaker identification system. Some modifications to the existing technique of MFCC for feature extraction are also suggested to improve the speaker recognition efficiency.
\end{abstract}

Keywords : FPGA, NIOSII , Artificial Neural Network (ANN), Mel frequency cepstral coefficients (MFCC), Speaker recognition 